\title{
Presignaled behavior as a predictor of signaled DRL performance
}

\author{
HENRY MARCUCELLA, JAMES S. MacDONALL, ILSE MUNRO, \\ and VICTORIA MOSELEY \\ Boston University, Boston, Massachusetts 02215
}

\begin{abstract}
The purpose of the present experiment was to demonstrate that the pattern of responding generated by an unsignaled differential-reinforcement-of-low-rate schedule (DRL) is a good predictor of the degree of stimulus control obtained when discriminative stimuli are introduced. The responses of 14 rats were first reinforced on an unsignaled DRL 25-sec schedule for 11 sessions. On Session 12 the schedule was changed to a signaled DRL 25-sec schedule for 11 sessions. The degree of stimulus control acquired by the added discriminative stimuli. was inversely related to the efficiency ratio calculated from the unsignaled performance.
\end{abstract}

Stimulus control of responding is typically established by (1) presenting two stimuli to an organism, either simultaneously or successively, and (2) reinforcing responding in the presence of one stimulus but not the other. However, the extent to which responding comes under the control of the stimulus properties associated with periods of reinforcement (S1) and nonreinforcement (S2) also depends upon other features of the discrimination procedure. These other features include (1) the physical relation of the response to stimulus site (Harrison, Downey, Segal, \& Howe, 1971; Jarvik, 1953, 1956; Nissen \& Jenkins, 1943; Stollnitz \& Schrier, 1962); (2) the discriminative function of reinforcing stimuli (Jenkins, 1965); and (3) the discriminative function of the organism's own behavior (Blough, 1963).

The degree of stimulus control acquired by discriminative stimuli may also depend upon the temporal and sequential programming of stimulus conditions. Marcucella (1974) demonstrated that the degree of control exerted by the discriminative stimuli of a signaled DRL schedule was inversely related to the DRL value. At short DRL values, time appeared to govern responding; many responses were emitted in $\mathrm{S} 2$ and the latency of the first response to the onset of $\mathrm{S} 1$ varied considerably. In short, performance resembled that obtained with unsignaled DRL schedules. At long DRL values, $\mathrm{S} 1$ and $\mathrm{S} 2$ acquired complete control of responding. At intermediate DRL values, an intermediate degree of stimulus control was acquired by $\mathrm{S} 1$ and $\mathrm{S} 2$. However, at any given intermediate $\mathrm{DRL}$ value, the degree of control acquired by $\mathrm{S} 1$ and $\mathrm{S} 2$ varied considerably between subjects.

Reprints may be obtained from Henry Marcucella, Department of Psychology, Boston University, 64 Cummington Street, Boston, Massachusetts 02215. James S. MacDonall is now at the Washingtonian Center for Addictions, 41 Morton Street, Boston, Massachusetts 02130.
This between-subjects variability suggested that during the acquisition of stimulus control, when both temporal and discriminative stimuli were available, the degree of control acquired by $\mathrm{S} 1$ and $\mathrm{S} 2$ was related to the relative strength of the concurrent temporal cues. Thus, when temporal and discriminative stimuli were both present, temporal cues may have exerted greater control of responding of one subject, while the discriminative stimuli may have controlled responding of another. This analysis suggested that the pattern of responding controlled by the temporal cues in the absence of $\mathrm{S} 1$ and $\mathrm{S} 2$ should be a good predictor of the degree of control acquired by $\mathrm{S} 1$ and $\mathrm{S} 2$. The present experiment demonstrated that the degree of stimulus control acquired when $S 1$ and $S 2$ were added to a DRL 25-sec schedule after stable unsignaled performance was obtained was inversely related to the efficiency ratio of the unsignaled performance.

\section{METHOD}

\section{Subjects}

Fifteen Sprague Dawley male albino rats, approximately 200 days old, were maintained at $85 \%$ of their free-feeding weight throughout the experiment. Water was available only in the home cage.

Prior to this experiment, all rats were fed ad lib for 90 days. Seven rats were fed a standard Purina Lab Chow diet, four rats a nutritionally adequate (Freund \& Walker, 1971) Metracalsucrose liquid diet, and four rats alternated between the lab chow and Metracal-sucrose liquid diet. Other data suggest that the Metracal-sucrose liquid diet has no effect on conditioning (Freund, 1970; Freund \& Walker, 1971; Walker \& Freund, 1971, 1973). At the end of this 90-day special feeding period, all rats were given unlimited access to lab chow and water for 2 weeks and then, during the next week, reduced to $85 \%$ of the resulting free-feeding weight.

\section{Apparatus}

Four animal chambers, each located in a sound-attenuating enclosure, were used in the experiment. The dimensions of three chambers were $23 \times 21 \times 19 \mathrm{~cm}$, and the fourth was $32 \times 21 \times$ $19 \mathrm{~cm}$. A standard Gerbrands rat lever, requiring an operating force of $.065 \mathrm{~N}$, was mounted in the middle of one wall of each 
chamber, $7 \mathrm{~cm}$ on center from the chamber floor. A $4 \times 4 \mathrm{~cm}$ opening for a Gerbrands food cup was located on the same wall, $7 \mathrm{~cm}$ to the left and $4 \mathrm{~cm}$, on center, below the rat lever. Reinforcers were 45-mg Noyes food pellets.

Two 28-V heavy-duty relays were located on either side of the animal enclosure, $15 \mathrm{~cm}$ from the lever and in line with the front wall of the chamber. The loci of the clicking noise generated by the rapid operation of the relays $(10$ times/sec) were used as the discriminative stimuli. Solid state and electromechanical recording and scheduling equipment were located in and adjacent room.

\section{Procedure}

All animals were first leverpress trained. After the first 30 responses were reinforced, responses of all subjects were reinforced on an unsignaled DRL 25-sec schedule for 11 sessions. Responses were reinforced only if the time from the previously emitted response exceeded $25 \mathrm{sec}$; responses that terminated interresponse times (IRT) less than $25 \mathrm{sec}$ were not reinforced but initiated a new IRT. Each session was terminated after 120 reinforcements. During unsignaled sessions, both clicking relays were operated continuously throughout the session. The houselight was on during the session and off before and after each session.

At the beginning of Session 12, the schedule was changed from unsignaled DRL $25-\mathrm{sec}$ to signaled DRL 25-sec. During the 11 sessions in which reinforcer availability was signaled, only one relay (S2) was operated when the reinforcer was not available. When the reinforcer became available, $\mathrm{S} 2$ was terminated and the other relay (S1) was operated. The first response after the onset of the $S 1$ resulted in the presentation of the reinforcer, the termination of S1, and the onset of S2. Thus, the temporal spacing of responses required for reinforcement, during signaled and unsignaled conditions, was identical with the exception that during signaled sessions external discriminative stimuli were introduced to signal when responses would or would not be reinforced.

\section{RESULTS}

Diet

Performances during the last five sessions of the unsignaled DRL condition were not related to diet

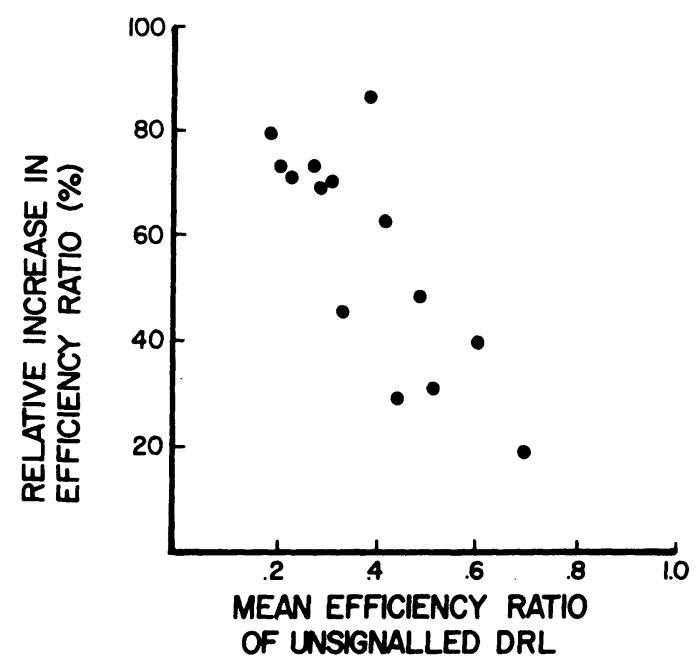

Figure 1. The relative increase in efficiency ratio produced by the introduction of the discriminative stimuli as a function of the efficiency ratio obtained during the last five sessions of the unsignaled condition. history. Instead, the efficiency ratios calculated from the performance of the animals of a particular diet group varied over a wide range.

\section{Stimulus Control}

Figure 1 shows, for each subject, the relative increase in the efficiency ratio produced by the introduction of the discriminative stimuli as a function of the efficiency ratio obtained during the last five sessions of unsignaled DRL $25 \mathrm{sec}$. The efficiency ratio was obtained by dividing the minimum possible time required to complete the session $(50 \mathrm{~min})$ by the obtained session time. In order to equate for the different levels of efficiency obtained by each subject during the unsignaled condition, each point in Figure 1 is expressed as a percentage of the maximum increase possible for that animal. For example, suppose for a particular animal the mean efficiency ratio for the last five sessions of the unsignaled condition was .7 and the mean efficiency ratio for the last five sessions of the signaled condition was .85 . Then the relative increase in efficiency would have been calculated by dividing the actual increase in the efficiency ratio, .15 , by the maximum possible increase in efficiency, .30 , and be equal to .50 .

Figure 1 shows that the percentage increase in the efficiency ratio, produced by the introduction of the discriminative stimuli, is inversely correlated with the mean efficiency ratio obtained during the last five sessions of the unsignaled condition. The Pearson product-moment correlation coefficient was calculated to be $-.77(\mathrm{p}<.01)$. Thus, the larger the efficiency ratio during the unsignaled condition, the smaller was the relative increase in the efficiency ratio when the discriminative stimuli were added.

Figure 2 shows the mean IRT distributions for both signaled (bottom) and unsignaled (top) conditions for both high- (left half) and low- (right half) efficiency animals. To determine high- and low-efficiency animals, all animals were first ranked in order of mean efficiency ratios obtained during the last five sessions of the unsignaled condition. The five most efficient animals were assigned to the high-efficiency group and the five least efficient animals to the low-efficiency group. Class interval size was equal to $5 \mathrm{sec}$. All IRTs falling into the 6th class interval or above were reinforced and all IRTs greater than $50 \mathrm{sec}$ were included in the 11 th class interval.

In Figure 2 we see that the primary difference between the IRT distribution of the high- and lowefficiency groups was the skewness of the distribution; the IRT distribution for the high-efficiency group was negatively skewed, while the IRT distribution for the low-efficiency group was positively skewed.

Although the introduction of the discriminative stimuli decreased the frequency of unreinforced IRTs of both groups, the stimuli had much less effect upon the reinforced IRT distribution of the high-efficiency animals; that is, even though $\mathrm{S} 1$ signaled reinforcer availability, the high-efficiency animals did not respond 


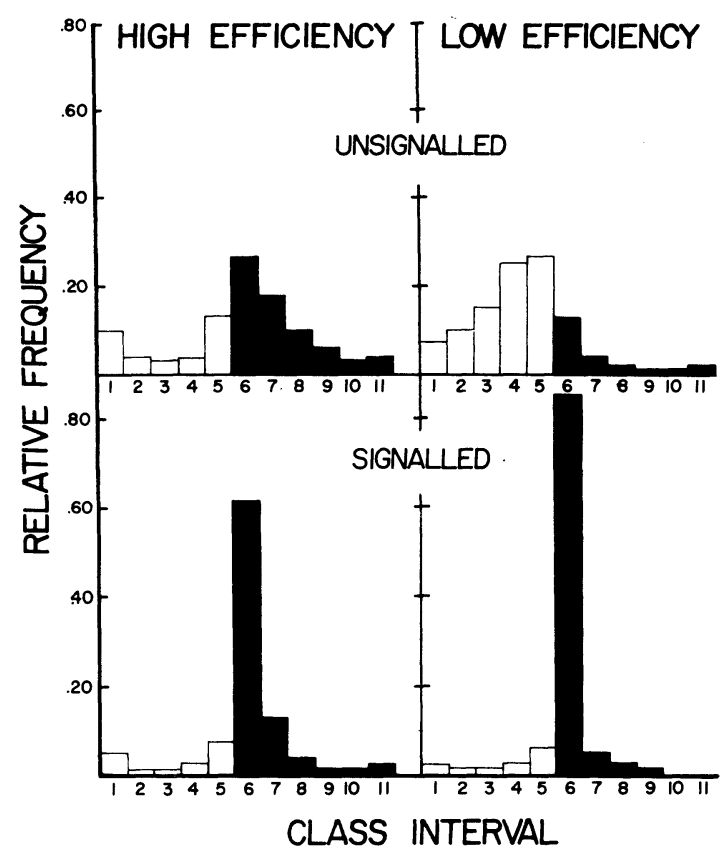

Figure 2. Mean interresponse time (IRT) distributions for both signaled and unsignaled conditions for both high- and lowefficiency subjects.

immediately following the onset of $\mathrm{S} 1$. Thus, the relative frequency of IRTs falling into the seventh class interval or above remained quite high. In contrast, the lowefficiency animals terminated most reinforced IRTs immediately following the onset of $\mathrm{S} 1$. As a result, the relative increase in efficiency ratio, which is sensitive to errors of over- as well as underestimation, was greater for those subjects that were responding less efficiently prior to the introduction of the stimuli. The absolute increase in efficiency was also greater for the subjects that were responding less efficiently during the unsignaled condition. Thus, the subjects that were initially responding more efficiently became the less efficient subjects following the introduction of the stimuli.

\section{DISCUSSION}

The results of the present experiment demonstrated that, although the introduction of discriminative stimuli improved the discriminative performance of all subjects, the degree of improvement was inversely related to the degree of control exerted by temporal cues prior to the introduction of the stimuli.

Marcucella (1974) suggested that during acquisition the effect of signaling reinforcer availability in DRL schedules was a function of the DRL value. At short DRL values, time, a highly salient stimulus for rats, governs responding even though the use of the available discriminative stimuli would increase the obtained rate of reinforcement. At long DRL values, where the potential loss of reinforcement is much greater, rats shift to exteroceptive stimulus cues.
A similar explanation is much more difficult to apply to the results of the present experiment, where stable behavior on unsignaled DRL 25-sec schedules was obtained prior to the introduction of the discriminative stimuli. For example, although the increase in reinforcement rate was greater for lowefficiency animals, one is left with the problem of explaining how rats could have discriminated a potential increase in reinforcement rate; that is, the rats shifted to exteroceptive control and, as a result, reinforcement rate increased. Such an interpretation requires either the assumption that rats actively sample the utility of available cues to reinforcement or the assumption of a critical reinforcement frequency below which rats will shift from temporal to exteroceptive cues.

A simpler explanation is that the effect on performance of adding stimuli to unsignaled DRL schedules is only an indirect result of the DRL value. The primary determiner of signaled performance may be the pattern of behavior occurring at the time of the schedule change. For example, if many unreinforced responses are occurring during the unsignaled condition at the time of the stimulus change, these responses will be emitted in the presence of an extinction-correlated stimulus (S2) and delay the onset of $\mathrm{S} 1$ for a considerable period of time. This procedure is identical to most discrimination procedures where responding is extinguished in the presence of one stimulus and reinforced in the presence of another. The data of the present experiment support this analysis. For example, during the first session following the introduction of the stimuli, the five least efficient animals made at least four times the number of $S 2$ responses and remained in the presence of S2 twice as long as the five most efficient animals. In addition, most of these unreinforced S2 responses occurred during the first several $S 2$ intervals.

Thus, it seems that the effect of the DRL value occurs through its indirect effect upon the pattern of responding. For example, if increasing the DRL value leads to more IRTs shorter than the minimum reinforced IRT, then an added signal should exert strong control over responding. It should be noted that, according to this interpretation, not all increases in DRL value necessarily lead to greater stimulus control. If an animal, even at large DRL values, emitted few unreinforced responses prior to the introduction of the discriminative stimuli, then the behavior of the animal would not make contact with the stimuli when they were introduced. The animal would continue to respond, at least in part, on the basis of the temporal cues produced by the schedule.

The advantage of this interpretation is that one need not assume that rats possess an internal mechanism that allows them to either sense or test differences in reinforcement rate. Instead, the shift from temporal to exteroceptive cues and vice versa may simply be a function of the ratio of unreinforced to reinforced IRTs occurring in the unsignaled condition. It may be that if this ratio remains below a certain level then temporal cues will continue to control behavior simply because the behavior fails to make contact with the added stimuli and their associated contingencies of reinforcement.

\section{REFERENCES}

Blough, D. S. Interresponse time as a function of variables: A new method and some data. Journal of the Experimental Analysis of Behavior, 1963, 6, 237-246.

FrEUND, G. Impairment of shock avoidance learning after long-term alcohol ingestion in mice. Science, 1970, 168. 1599-1601.

Freund, G., \& Walker, D. W. Impairment of avoidance learning by prolonged ethanol consumption in mice. Journal of Pharmacology and Experimental Therapeutics. 1971. 179. 284-292. 
Harrison, J. M., Downey, P., Segal, M., \& Howe, M. Control of responding by the location of auditory stimuli: Rapid acquisition in monkeys and rats. Journal of the Experimental Analysis of Behavior, 1971, 15, 379-386.

JARVIK, M. E. Discrimination of colored food and food sign by primates. Journal of Comparative and Physiological Psychology, 1953, 46, 390-392.

JARVIK. M. E. Simple color discrimination in chimpanzees. Journal of Comparative and Physiological Psychology, 1956, 49, 492-495.

Jenkins, H. M. Measurement of stimulus control during discriminative operant conditioning. Psychological Bulletin, 1965, 64, 365-376.

Marcucella, H. Signalled reinforcement in differential reinforcement-of-low-rate schedules. Journal of the Experimental Analysis of Behavior, 1974, 22, 381-390.

Nissen, H. W., \& Jenkins, W. D. Reduction and rivalry of cues in the discrimination behavior of chimpanzees. Journal of Comparative Physiology, 1943, 35, 85-95.

Stollnitz, F., \& Schrier, A. M. Discrimination learning by monkeys with spatial separation of cue and response. Journal of Comparative and Physiological Psychology, 1962, 55, 876-881.

WALKeR, D. W., \& Freund, G. Impairment of shuttle box avoidance learning following prolonged alcohol consumption in rats. Physiology and Behavior, 1971, 7, 773-778.

Walker, D. W., \& Freund, G. Impairment of timing behavior after prolonged alcohol consumption in rats. Science, 1973, 182, 597-599.

(Received for publication June 22, 1977.) 